\title{
Intracranial aneurysm wall enhancement: fact or fiction?
}

\section{René van den Berg ${ }^{1}$}

Published online: 21 January 2020

(C) Springer-Verlag GmbH Germany, part of Springer Nature 2020

In the quest for a better understanding of the "behavior" of intracranial aneurysms, research activities have shifted from computational fluid dynamics towards imaging-based support for inflammatory processes in the aneurysm wall as a possible underlying cause for aneurysm rupture or instability. One of the landmark papers on the relation of inflammatory processes in the aneurysm wall and the rupture risk is by Frösen et al. [1]. Histological aneurysm wall analysis was performed in ruptured and unruptured aneurysms focusing on cellular mechanisms of adaptation to increased hemodynamic stress, which are partly controlled by cytokines released by inflammatory cells infiltrating the vascular wall. The idea of using vessel wall imaging (VWI) to visualize aneurysm wall enhancement links enhancement of the aneurysm wall to the local histological inflammatory process.

But a critical appraisal is warranted. In the Frösen paper, the most thinly lined aneurysms had the highest percentage of rupture. Aneurysm walls measure on average between 0.02 and $0.50 \mathrm{~mm}$. However, depicting aneurysm walls closer to the $0.02 \mathrm{~mm}$ end of the spectrum at $3 \mathrm{~T}$ is very difficult, as the image resolution of $0.8 \mathrm{~mm}$ is insufficient. Even at $7 \mathrm{~T}$, visualization is almost impossible. Pulsation artifacts degrade image quality and falsely increase wall thickness, requiring cardiac gating. Additionally, there are difficulties in reliable assessment of the aneurysm wall close to the brain parenchyma and, on top of that, intraluminal thrombus may hinder reliable assessment of wall thickness [2].

Nevertheless, aneurysms do show enhancement using VWI techniques, so the question remains: what is it that enhances? Although VWI technique is based on suppression of intraluminal signal, generating "black blood," this suppression depends on local flow conditions. A recent report using an aneurysm containing silicon model showed that at slow flow

René van den Berg

r.vdberg@amsterdamumc.nl

1 Department of Radiology and Nuclear Medicine, Amsterdam University Medical Centers, PO Box 9600, 1105 AZ Amsterdam, the Netherlands conditions, even with the best suppression techniques, enhancement close to the wall is seen, but this should be considered "pseudo" enhancement as the silicon model wall cannot enhance [3]. In vivo, one can expect these slow or turbulent flow conditions in larger, multi-lobulated aneurysms and aneurysms with blebs. These morphological characteristics are known to be related to an increased rupture risk and, therefore, this "pseudo" enhancement on VWI might simply reflect aneurysm morphology. True wall enhancement can be seen in large and giant aneurysms as these aneurysms exhibit a thickened wall, in which the development of an enhancing vasa vasorum is acknowledged.

Findings of aneurysm wall enhancement should therefore be interpreted with caution. In earlier reports, Matouk et al. could differentiate ruptured from unruptured aneurysm in patients with multiple intracranial aneurysms presenting with a subarachnoid hemorrhage [4]. Subsequent publications from other investigators showed that unruptured aneurysms can also enhance and it was then argued that aneurysm wall enhancement could be used as an indirect marker of aneurysmal wall inflammation to estimate rupture risk or to predict an "unsteady aneurysm state." This enhancement was found to be related to conventional rupture-related risk factors (aneurysm size) and correlated to the PHASES score. Aneurysms with a score $>3$ were more likely to show enhancement and were therefore at a higher risk of rupture [5]. However, one should realize that rupture risk with a PHASES score of " 3 " is extremely low with a percentage of only 0.7 in five years! Unfortunately, then, both ruptured and unruptured aneurysms can show wall enhancement and the potential to differentiate ruptured from unruptured aneurysms no longer seems to hold true.

Recently, local hemodynamic conditions were linked to focal changes in the intracranial aneurysm wall and it was concluded that slow swirling flow and low oscillatory wall shear stress lead to atherosclerotic/hyperplastic changes and high flow conditions near impingement zone, characterized by higher wall shear stress, lead to local "thinning" of the wall [6]. It might be that these findings point to the indirect relation of flow conditions-aneurysm rupture risk-and 
inflammatory wall changes. More research on the topic is needed, with a better linkage to histopathological focal inflammatory changes with enhancement patterns on VWI in correlation with flow dynamics, in which the role of high or low (or the alternating force) wall shear stress can be elucidated. Unfortunately, the vast majority of aneurysms are typically treated endovascularly, impeding such progress.

Funding information NA

\section{Compliance with ethical standards}

Conflict of interest I declare that there is no conflict of interest.

Ethical approval This article does not contain any studies with human participants or animals performed by the author.

Informed consent NA

\section{References}

1. Frosen J, Piippo A, Paetau A, Kangasniemi M, Niemela M, Hernesniemi J et al (2004) Remodeling of saccular cerebral artery aneurysm wall is associated with rupture: histological analysis of 24 unruptured and 42 ruptured cases. Stroke. 35(10):2287-2293
2. Kleinloog R, Korkmaz E, Zwanenburg JJ, Kuijf HJ, Visser F, Blankena R, Post JA, Ruigrok YM, Luijten PR, Regli L, Rinkel GJ, Verweij BH (2014) Visualization of the aneurysm wall: a 7.0Tesla magnetic resonance imaging study. Neurosurgery. 75(6):614 622 discussion 22

3. Cornelissen BMW, Leemans EL, Coolen BF, Peper ES, van den Berg R, Marquering HA, Slump CH, Majoie CBLM (2019) Insufficient slow-flow suppression mimicking aneurysm wall enhancement in magnetic resonance vessel wall imaging: a phantom study. Neurosurg Focus 47(1):E19

4. Matouk CC, Mandell DM, Gunel M, Bulsara KR, Malhotra A, Hebert R et al (2013) Vessel wall magnetic resonance imaging identifies the site of rupture in patients with multiple intracranial aneurysms: proof of principle. Neurosurgery. 72(3):492-496 discussion 6

5. Hartman JB, Watase H, Sun J, Hippe DS, Kim L, Levitt M, Sekhar L, Balu N, Hatsukami T, Yuan C, Mossa-Basha M (2019) Intracranial aneurysms at higher clinical risk for rupture demonstrate increased wall enhancement and thinning on multicontrast $3 \mathrm{D}$ vessel wall MRI. Br J Radiol 92(1096):20180950

6. Cebral JR, Detmer F, Chung BJ, Choque-Velasquez J, Rezai B, Lehto H, Tulamo R, Hernesniemi J, Niemela M, Yu A, Williamson R, Aziz K, Shakur S, Amin-Hanjani S, Charbel F, Tobe Y, Robertson A, Frösen J (2019) Local hemodynamic conditions associated with focal changes in the intracranial aneurysm wall. AJNR Am J Neuroradiol 40(3):510-516

Publisher's note Springer Nature remains neutral with regard to jurisdictional claims in published maps and institutional affiliations. 